\title{
Numerical Simulation and Flow Visualization Using Soap Film of the Self-Organized Vortex Structure in the Wake of an Array of Cylinders
}

\author{
Mohammad Rafiqul Haque, Mohamed Fayed, Amy-Lee Gunter, Othman Smadi, \\ Lyes Kadem and Hoi Dick $\mathrm{Ng}^{\dagger}$ \\ Department of Mechanical and Industrial Engineering, Concordia University, \\ 1455 de Maisonneuve Blvd. West, Montréal, Québec, H3G 1M8, Canada
}

\author{
${ }^{\dagger}$ Corresponding author \\ Department of Mechanical and Industrial Engineering \\ Concordia University \\ 1455 de Maisonneuve Blvd. W. \\ Montréal, QC, H3G 1M8 Canada \\ Tel: (514) 848-2424 ext. 3177 \\ Fax: (514) 848-3175 \\ †e-mail: hoing@encs.concordia.ca
}

Revised Portfolio paper submitted to Journal of Visualization

March 14, 2011 


\title{
Numerical Simulation and Flow Visualization Using Soap Film of the Self-Organized Vortex Structure in the Wake of an Array of Cylinders
}

\author{
Mohammad Rafiqul Haque, Mohamed Fayed, Amy-Lee Gunter, Othman Smadi, \\ Lyes Kadem and Hoi Dick $\mathrm{Ng}^{\dagger}$ \\ Department of Mechanical and Industrial Engineering, Concordia University \\ Montréal, QC, H3G 1M8 Canada \\ †e-mail: hoing@encs.concordia.ca
}

Wakes behind blunt bodies, such as a cylinder or square, are classical problems in fluid mechanics (e.g., Oertel 1990; Williamson 1996). The wake structures past the pillars of a bridge, or the wake behind a propelling ship are some real life examples. In this study, numerical simulations and flow visualizations have been performed to elucidate vortex interactions in the wake of an array of cylinders. The computational results are obtained by using both the two-dimensional unsteady laminar viscous model, and the Large-Eddy-Simulation (LES) model, with the Fluent ${ }^{\circledR}$ Software for Reynolds numbers corresponding to both laminar and turbulent flows, respectively. The spacing between neighboring cylinders is $S=w / D=4.0$, where $D$ is the diameter of the cylinders $(1.8 \mathrm{~mm})$ and $w$ is the distance between two cylinders' center. The computational domain $(5.2 \mathrm{~cm} \mathrm{x} 4.5 \mathrm{~cm})$ contains 340,654 cells and the computational time step for the simulations equals $d t=0.0050 \mathrm{sec}$. A uniform flow is imposed at the inlet boundary and a pressure outlet boundary for the flow outlet. The cylinders and side walls are modeled under no-slip boundary conditions (Huang et al. 2006).

The CFD results are presented in Fig. 1 showing the vorticity contours, which illustrate in detail the vortex dynamics of the cylinders' wake. For comparison, the wake structure present for the same configuration and approximately the same flow conditions (excluding the low Reynolds number laminar condition due to the limitation of the experimental techniques) is visualized using the soap-film method (Zhang et al. 2000) and photo results are presented in Fig. 2. A two-dimensional soap film tunnel is used, which consists of a test section of $1 \mathrm{~m}$ by $10 \mathrm{~cm}$ capable of producing mean flows between $2.70 \mathrm{~m} / \mathrm{s}$ to $6.50 \mathrm{~m} / \mathrm{s}$. The kinematic viscosity $v$, of the soap film at room temperature of $20^{\circ} \mathrm{C}$ is $1.004 \times 10^{-6} \mathrm{~m}^{2} / \mathrm{s}$. Once the array of obstacles is placed in the soap film, we make use the optical properties of the soap film which are visible in the presence of a low pressure sodium lamp (590 nm). To track and capture the wake dynamics of the flow motion, a high-speed pco.1200hs camera is used recording at a frame rate of $800 \mathrm{fps}$. The use of the present lighting system clearly highlights the interference patterns of the soap film, and illustrates how the wakes evolve in the flow.

For the low Reynolds number laminar regime considered in the numerical study (i.e., $R e=100$ ), regular wake pattern exists and manifests unsteadily into different forms. Overall, a well-organized wake structure is observed on both sides of the geometric middle of the six cylinders. However, in the case of high Reynolds number ( $R e \sim 7500)$, an increase of the free stream flow velocity causes a wake transition and the regularity breaks down, resulting in a chaotic flow pattern with turbulent mixing in the far-field wake. In our CFD simulation, it appears that the regular vortex pattern breaks at a Reynolds number of the order of $O(2000)$, for example, see Fig. 3. However, for the soapfilm experiments with $R e=4840$, similar large scale regular vortices structure is still observed in the far-wake. To explain this apparent lack of agreement, it is important to note that numerical simulations at increasing $R e$ are computationally challenging and required great attention, particularly at transitional Reynolds numbers, because of the sensitivity of the vortex shedding on the numerical details such as the discretization scheme, boundary condition, sub-grid scale modeling, grid resolution and time step, etc. (Breuer 1998). Simulation needs to run for very long enough time so that the result could approach physically meaningful solutions; this is computationally expensive. This requirement thus limits the present direct comparison and detailed computational analysis is further required.

In this portfolio, some interesting nonlinear instability modes and patterns developed in the wake of an array of cylinders are presented. The wake dynamics observed from both the numerical results, and the experimental flow visualization using flowing soap-film technique, are illustrated to be in agreement at least qualitatively. From the point of view of a dynamical process, the formation of these wake patterns from multiple cylinders appears to be a bifurcation phenomenon (Hourigan et al. 2007); and similar to the case of flow past a single blunt body, further detailed investigation is needed in view of global stability and bifurcation to identify different modes and the critical Reynolds number for the instability transition. Furthermore, from the physical consideration, the present results perhaps appear to suggest that the mechanism of the breakdown of the large scale regular vortex pattern at higher Reynolds number may resemble to that of large-scale vortex dislocation (Williamson 1996).

\section{Acknowledgment}

This work is supported by the Natural Sciences and Engineering Research Council of Canada (NSERC). 


\section{References}

Breuer M. (1998) Large eddy simulation of the sub-critical flow past a circular cylinder: numerical and modeling aspects. Int. J. Numer. Meth. Fluids, 28:1281-1302.

Hourigan K, Thompson MC, Sheard GJ, Ryan K, Leontini JS and Johnson SA (2007) Low Reynolds number instabilities and transitions in bluff body wakes. J. Phys.: Conf. Ser. 64012018.

Huang Z, Olson JA, Kerekes RJ and Green SI (2006) Numerical simulation of the flow around rows of cylinders. Computers Fluids 35:485-491.

Oertel H (1990) Wakes behind blunt bodies. Ann Rev Fluid Mech 22:539-562.

Williamson CHK (1996) Vortex dynamics in the cylinder wake. Ann Rev Fluid Mech. 28:477-539.

Zhang J, Childress S, Libchaber A and Shelly MJ (2000) Flexible filaments in a flowing soap film as a model for one-dimensional flags in a two-dimensional wind. Nature 408: 835-839.
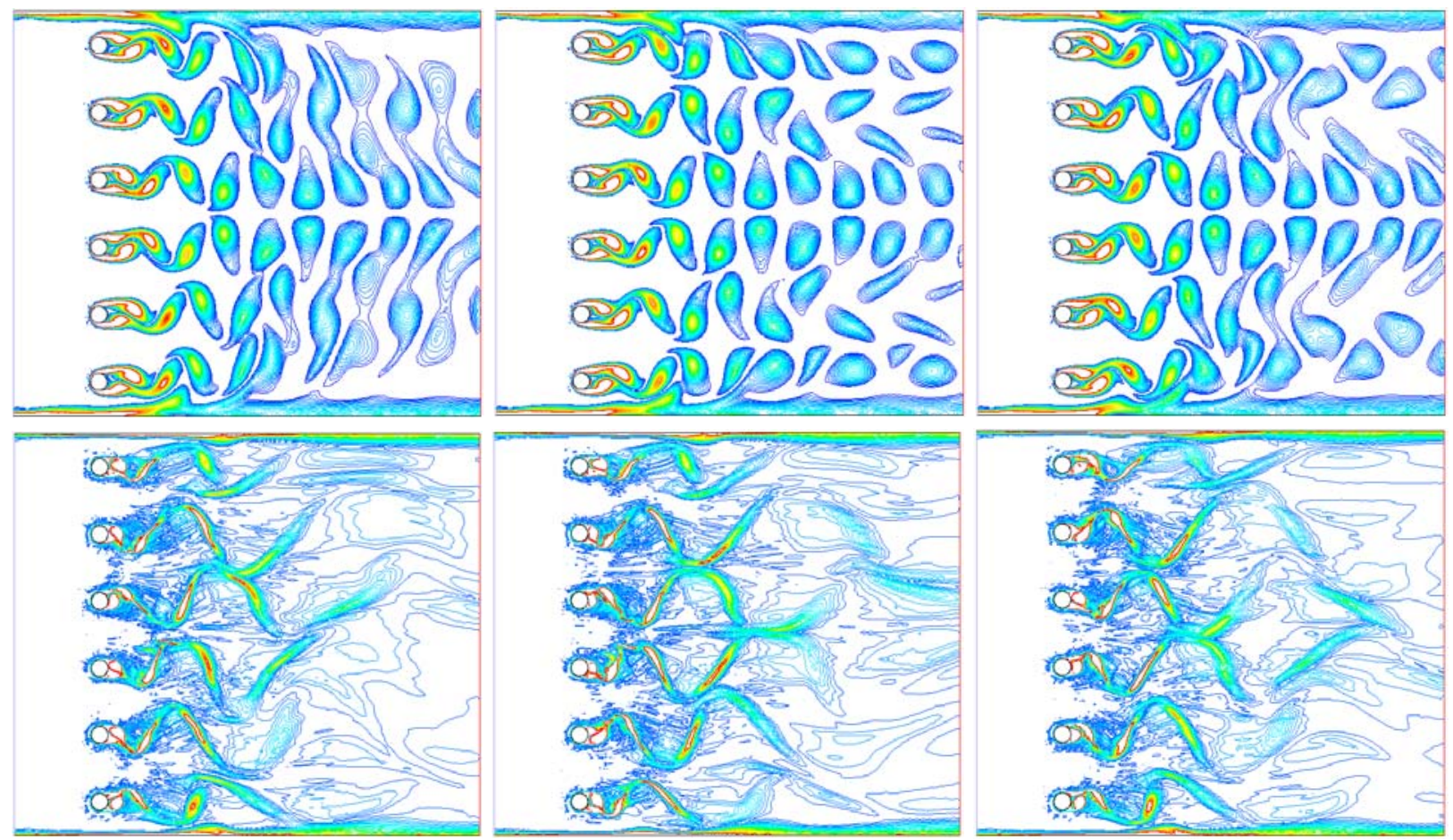

Figure 1. Images illustrating the vortex dynamics in the wake of an array of six cylinders at three different instant times. Top) Numerical simulations for $R e=100$ using the laminar viscous model; and Bottom) for $R e=7500$ using the LES model. 

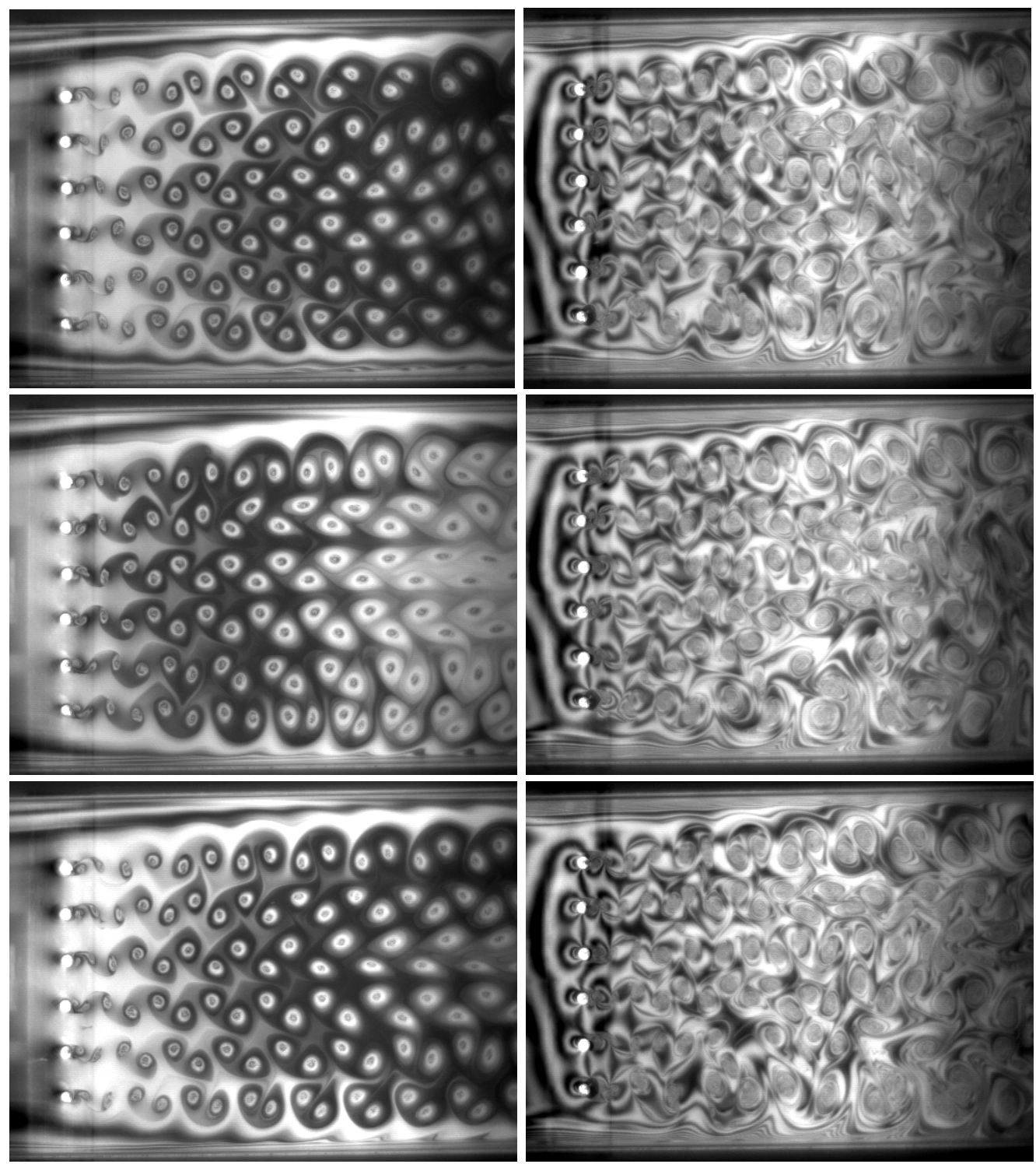

Figure 2. Flow visualization using a flowing two-dimensional soap film, illustrating the vortex dynamics in the wake of an array of six cylinders at three different instant times with flow speed of $2.7 \mathrm{~m} / \mathrm{s}, R e=4840$ (left) and 4.2 $\mathrm{m} / \mathrm{s}, \operatorname{Re}=7530$ (right).

a)

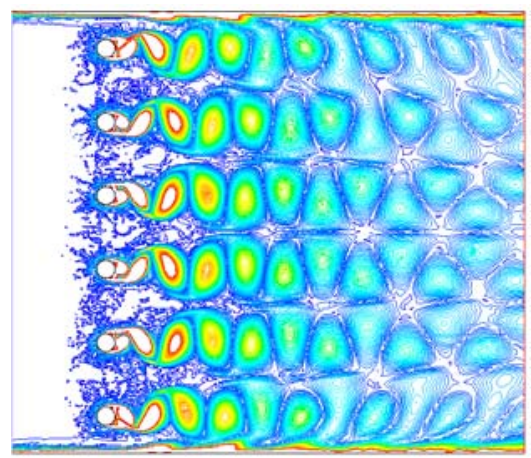

b)

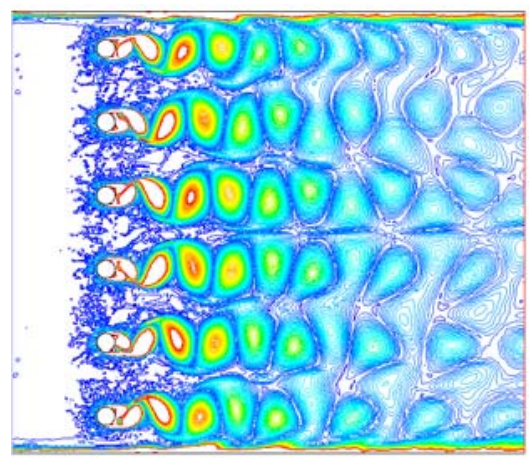

Figure 3. LES simulations of the wake of an array of six cylinders for $\mathbf{a}) \operatorname{Re}=1687$; and $\mathbf{b}) \operatorname{Re}=1875$. 